\title{
One-loop corrections to the Drell-Yan process in SANC: the charged current case
}

\author{
A. Arbuzov ${ }^{1, a}$, D. Bardin ${ }^{2}$, S. Bondarenko ${ }^{1}$, P. Christova ${ }^{2}$, L. Kalinovskaya ${ }^{2}$, G. Nanava ${ }^{2}$, R. Sadykov $^{2}$ \\ 1 BLTPh, JINR, Dubna, 141980, Russia \\ 2 DLNP, JINR, Dubna, 141980, Russia
}

Received: 29 December 2006 /

Published online: 17 February 2007 - (C) Springer-Verlag / Società Italiana di Fisica 2007

\section{Erratum to:}

\section{Eur. Phys. J. C DOI 10.1140/epjc/s2006-02505-y}

Sect. 4 contains an incorrect and misleading consideration of a double-differential cross section. Moreover, the formulae given there do not correspond to the ones used in our computer codes. The section should read as follows.

The differential cross section of the Drell-Yan process can be obtained from the convolution of the partonic cross section with the quark density functions:

$$
\begin{array}{r}
\frac{\mathrm{d} \sigma_{\mathrm{RC}}^{p p \rightarrow \mu^{+} \nu X}(s, c)}{\mathrm{d} c}=\sum_{q_{1} q_{2}} \int_{0}^{1} \int_{0}^{1} \mathrm{~d} x_{1} \mathrm{~d} x_{2} \bar{q}_{1}\left(x_{1}, M^{2}\right) \\
\times \bar{q}_{2}\left(x_{2}, M^{2}\right) \frac{\mathrm{d} \hat{\sigma}^{q_{1} q_{2} \rightarrow \mu^{+} \nu}(\hat{s}, \hat{c})}{\mathrm{d} \hat{c}} \mathcal{J} \Theta\left(c, x_{1}, x_{2}\right),
\end{array}
$$

The online version of the original article can be found at http://dx.doi.org/10.1140/epjc/s2006-02505-y

\footnotetext{
a e-mail: arbuzov@thsun1.jinr.ru
}

where the parton densities with bars mean the ones modified by the subtraction of the quark mass singularities; the step function $\Theta\left(c, x_{1}, x_{2}\right)$ defines the phase space domain corresponding to the given event selection procedure. The partonic cross section is taken in the centerof-mass reference frame of the initial quarks, where the cosine of the muon scattering angle, $\hat{c}$ is defined. The transformation into the observable variable $c$ involves the Jacobian:

$$
\begin{aligned}
& \mathcal{J}=\frac{\partial \hat{c}}{\partial c}=\frac{4 x_{1} x_{2}}{a^{2}}, \quad a=x_{1}+x_{2}-c\left(x_{1}-x_{2}\right), \\
& \hat{c}=1-(1-c) \frac{2 x_{1}}{a}, \quad \hat{s}=s x_{1} x_{2} .
\end{aligned}
$$

An analogous formula can be written for any other choice of a differential distribution as well as for the total cross section. 